\title{
Avaliação do Nível de Alfabetização Computacional de Indivíduos Egressos do Ensino Médio: Uma Revisão Sistemática da Literatura
}

\author{
Williane Rodrigues de Almeida Silva ${ }^{1}$, Edmir Parada Vasques Prado $^{1}$ \\ ${ }^{1}$ Universidade de São Paulo (USP) \\ Av. Arlindo Bettio, 1000 - CEP 03828-000 - São Paulo - SP - Brasil \\ \{williane.rodrigues, eprado\}@usp.br
}

\begin{abstract}
Various are the facilities available to individuals enabled in the use of ICT. However, the relevance of this knowledge in academia and in the labour market, in which individuals are constantly called for the implementation of activities supported by computers and applications. Notes that the self-learning on ICT is restricted to a few specific aspects, and considered insufficient for a minimally adequate preparation. To identify studies related to instruments that assess the degree of knowledge and skills related to Computer Literacy, a systematic review of the literature.
\end{abstract}

Resumo. Diversas são as facilidades disponibilizadas para os indivíduos habilitados no uso da TIC. Entretanto, destaca-se a relevância dessa habilitação no ambiente acadêmico e no mercado de trabalho, no qual os individuos são constantemente chamados à execução de atividades apoiadas por computadores e aplicativos. Constata-se que a autoaprendizagem sobre TIC é restrita a alguns aspectos especificos, e considerada insuficiente para um preparo minimamente adequado. Com o objetivo de identificar estudos relacionados a instrumentos que avaliam o grau de conhecimentos $e$ habilidades relacionados a Alfabetização Computacional, realizou-se uma Revisão Sistemática da Literatura.

\section{Introdução}

O uso diário dos computadores e o acesso à internet é algo comum nos dias atuais. Empresas e órgãos governamentais de diversos países estão cada vez mais dependentes de sistemas de tecnologia de informação e comunicação (TIC) [Wang e Prado 2015]. Como consequência, o cidadão tem que usar os mais variados recursos disponibilizados pelas TICs, visando melhorar seu desempenho pessoal e profissional [Coutinho e Lisbôa 2011].

A TIC tem influenciado bastante no processo de aprendizagem. O método tradicional de ensino praticado por décadas, baseado em papel, lápis e livros, não pode se manter alheio ou distante à existência da internet, dado o grande volume de informações úteis para o ensino de áreas distintas de conhecimento nos diversos níveis que essa ferramenta oferece [Gupta 2006].

Aprender conceitos relacionados a TIC, desenvolvendo habilidades e conhecimento a respeito do uso de computadores, tem se tornado importante na 
sociedade. Liao e Pope (2008) consideram que o conhecimento e as competências computacionais têm grande influência sobre uma nação, justificando que o impacto dos computadores na sociedade é amplo.

O acesso a infraestrutura mínima, no que diz respeito ao uso de computadores, englobando hardware e software, associado a um grau de conhecimento mínimo que os indivíduos devem ter em relação ao uso das TICs, tem sido destacado na literatura como uma condição essencial para a inclusão digital [Moura 2010]. A esse conjunto de conhecimentos e habilidades mínimas necessárias no uso das TICs para que um cidadão possa ser considerado incluído na sociedade digital denominou-se de Alfabetização Computacional (AC). Com isso, Filatro (2004) menciona que um processo de aquisição de habilidades básicas para o uso de computadores, redes e serviços de internet são ações da AC.

A partir disso, definiu-se uma questão de pesquisa principal para nortear este trabalho: Como avaliar o grau de Alfabetização Computacional (AC) de indivíduos que estão concluindo o ensino médio? A partir dessa, definiu-se duas questões de investigação mais específicas para a seleção dos estudos primários: (i) como medir o nível de AC de indivíduos? (ii) quais os instrumentos existentes para avaliar o grau de $\mathrm{AC}$ dos indivíduos?

Para responder as perguntas propostas, faz-se necessário uma Revisão Sistemática da Literatura (RSL) sobre trabalhos existentes visando consolidar os conhecimentos e habilidades relacionados à AC. O objetivo desta revisão é identificar estudos e pesquisas relacionados a instrumentos que avaliam o grau de conhecimentos e habilidades relacionados a AC de indivíduos, identificando as principais lacunas e oportunidades de pesquisa nesta área.

A fim de documentar os procedimentos executados e os resultados obtidos nesta RSL, este artigo está organizado da seguinte forma: na seção 2 menciona-se o método utilizado, bem como os passos utilizados na RSL, que ajudam a definir o tema proposto; na seção 3, os resultados e análises são discutidos; e finalmente, na seção 4 as considerações finais são apresentadas seguidas das referências bibliográficas.

\section{Método}

Segundo Kitchenham (2007 apud Aureliano e Tedesco, 2012), a Revisão Sistemática da Literatura (RSL) destina-se a identificar, avaliar e interpretar as pesquisas disponíveis relacionadas a um tema específico. Com o intuito de aplicar a RSL, tem-se a construção do protocolo de pesquisa, que é um documento composto por um conjunto de diretrizes que irão nortear a execução da pesquisa. Inicia-se esse protocolo com a determinação de questões de pesquisa. A partir dessas questões, fez-se o levantamento dos dados necessários para responde-las, orientando assim a leitura e seleção dos artigos. Essa seleção é realizada a partir de critérios de inclusão e exclusão pré-definidos. Por fim, elabora-se a síntese dos resultados e avaliação crítica do estudo.

\subsection{Questões de pesquisa}

Para realizar esta RSL, definiu-se uma questão de pesquisa principal: Como avaliar o grau de $A C$ de indivíduos que estão concluindo o ensino médio? Em seguida definiu-se 
VI Congresso Brasileiro de Informática na Educação (CBIE 2017)

Anais do XXIII Workshop de Informática na Escola (WIE 2017)

duas questões de investigação mais específicas que nortearam a seleção dos estudos primários analisados, respondendo assim à questão principal:

- QP1. Como medir o nível de AC de indivíduos?

Essa questão visa identificar como é medido o grau de AC de indivíduos, se por meio de navegadores, velocidade de digitação, entre outros. Assim, essa questão procura saber como é feito essa medição.

- QP2. Quais os instrumentos existentes para avaliar o grau de AC dos indivíduos?

O objetivo dessa questão é identificar quais instrumentos estão sendo utilizados para avaliar o grau de AC de indivíduos, como por exemplo, software, questionários, entre outros.

\subsection{Termos-chave da pesquisa}

A partir das estruturas das questões de investigação definidas anteriormente, foram identificados os principais termos (Alfabetização Computacional, TIC, Tecnologia da Informação e Comunicação, Inclusão Digital). Após essa identificação, tem-se a realização da tradução desses termos para o inglês (Computer Literacy, ICT, Information and Communication Technology, Digital Inclusion), por ser a língua mais utilizada nas bases de dados eletrônicas pesquisadas.

Os termos-chave foram pesquisados de duas formas: em uma das bases utiliza-se o caractere asterisco $(*)$, permitindo a variação de palavras que façam o uso desse símbolo; mas como a outra base de dados utilizada tem formas diferentes de elaborar sua string de busca, não foi possível fazer o uso do asterisco $\left(^{*}\right)$, por não aceitar esse formato nas palavras utilizadas, mantendo diferença entre estas.

\subsection{String de busca}

Segundo Costa (2010), as strings de busca são geradas a partir das estruturas das questões e da combinação dos termos-chave e sinônimos. São usados os conectivos OR (ou) entre os sinônimos identificados e AND (e) entre os termos-chave. Algumas adaptações são necessárias de acordo com as necessidades específicas de cada base de dados. Possíveis peculiaridades das bibliotecas digitais e adaptações mediante a isso são registradas. Desta forma, a string final utilizada nas bases de dados foram:

- String (Scopus), retornando 173 artigos: (TITLE-ABS-KEY("comput* litera*") AND (ict OR ICT OR informat* and communic* technol* OR digital inclu*) AND (comput* literacy OR digital inclu*) AND (computer literacy OR informa* and communica* technol*)). AND (LIMIT-TO(DOCTYPE,"ar") OR LIMIT-TO(DOCTYPE,"cp") OR LIMIT-TO(DOCTYPE,"ip")) AND (LIMITTO(SUBJAREA,"SOCI") OR LIMIT-TO(SUBJAREA,"COMP" ))

- String (ERIC), retornando 18 artigos: Title-abstract("computer liberal") AND (it OR it OR informal and community technol OR digital include) AND (computer literacy OR digital include) AND (computer literacy OR informal and community technol)) 
VI Congresso Brasileiro de Informática na Educação (CBIE 2017)

Anais do XXIII Workshop de Informática na Escola (WIE 2017)

\subsection{Fontes de busca}

Os critérios para a seleção das fontes foram: (i) disponibilidade de consulta aos artigos na web, cujos materiais contidos nelas sejam acessíveis; (ii) presença de mecanismos de busca usando palavras-chave; e, (iii) importância e relevância das fontes.

Assim como a string de busca definida, as bases de dados utilizadas foram: Scopus (https://www.scopus.com/), e ERIC (Education Resource Information Center) (http://eric.ed.gov/).

Definidos os estudos primários, os artigos selecionados foram lidos na íntegra, elaborando um resumo de cada um desses, destacando os pontos importantes e os resultados obtidos. Os trabalhos com pouca relevância para esta pesquisa foram descartados. A seguir são apresentados os critérios de inclusão e exclusão definidos para análise dos artigos selecionados.

\subsection{Seleção dos estudos e critérios (inclusão e exclusão)}

Os artigos encontrados foram selecionados por meio da verificação dos critérios de inclusão e exclusão estabelecidos; tal verificação executa-se mediante a análise dos títulos e abstracts dos artigos. Todos os artigos selecionados dos estudos primários foram lidos na íntegra e elaborando um resumo de cada um desses, destacando cada ponto importante e os resultados obtidos.

A inclusão de um trabalho é designada pela sua relevância em relação às questões de pesquisa, definidas ao analisar o título (tittle) e resumo (abstract) de cada artigo. Foram definidos os critérios de inclusão expostos no quadro a seguir:

Quadro 1. Critérios de Inclusão

\begin{tabular}{|l|l|}
\hline \multicolumn{2}{|c|}{ Critérios de Inclusão } \\
\hline CI 1 & Artigos disponíveis na web. \\
\hline CI 2 & $\begin{array}{l}\text { Artigos que apresentam palavras da string de busca no seu título ou } \\
\text { no seu resumo. }\end{array}$ \\
\hline CI 3 & Artigos escritos em inglês ou português. \\
\hline
\end{tabular}

Assim como nos critérios de inclusão, a partir da análise do título e resumo, são excluídos os estudos que se enquadrem em qualquer um dos casos expostos no quadro 2:

\section{Quadro 2. Critérios de Exclusão}

\section{Critérios de Exclusão}

CE 1 Artigos que trabalhem a AC para a formação de professores.

CE 2 Artigos que tratem a AC para crianças ou idosos.

CE 3 Artigos sobre a $\mathrm{AC}$ no ensino à distância. 
VI Congresso Brasileiro de Informática na Educação (CBIE 2017)

Anais do XXIII Workshop de Informática na Escola (WIE 2017)

\subsection{Processo de seleção dos estudos}

Após definir as questões de pesquisa, a string de busca foi submetida na base de dados Scopus, na qual foram encontrados 173 estudos, em seguida foram analisados por meio dos critérios de inclusão e exclusão, reduzindo para o total de 10 artigos, atendendo aos critérios selecionados. Já na base de dados ERIC, foram encontrados 18 estudos, mas, ao passar pelos critérios de inclusão e exclusão, apenas 3 destes foram considerados. Com isso, tem-se um total de 13 artigos considerados nesta revisão sistemática. A figura 1 apresenta os resultados produzidos pela relação de estudos primários aceitos e rejeitados neste trabalho.

Para quantificar e controlar a quantidade de trabalhos à medida que foram selecionados, criou-se no Microsoft (MS) Word uma tabela com os critérios de inclusão e exclusão, e para cada artigo analisado elaborou-se uma justificativa, afirmando se seria incluído ou excluído, se os critérios fossem atendidos, ia para a lista de critérios incluídos, senão, ia para a lista dos excluídos, mantendo assim uma organização entre estes.

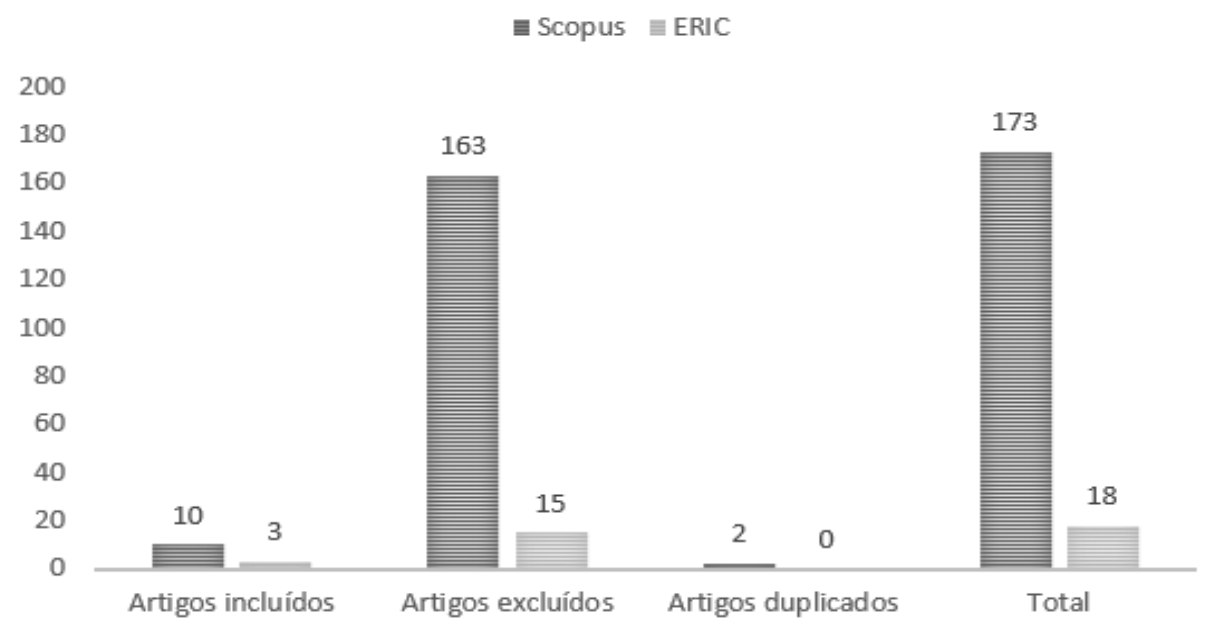

Figura 1. Seleção dos estudos primários (Scopus e ERIC)

Foram considerados apenas trabalhos que estavam de acordo com os critérios estabelecidos, e sempre verificando a relação destes com o tema da revisão. Segue no quadro 3 a lista dos artigos considerados no Scopus:

Quadro 3. Artigos considerados no Scopus

\begin{tabular}{|c|l|}
\hline $\mathbf{N}^{\mathbf{0}}$ & \multicolumn{1}{c|}{ Artigos } \\
\hline 1 & Measuring computer Literacy without questionnaires. \\
\hline 2 & Factorial invariance across gender of a perceived ICT literacy scale. \\
\hline 3 & $\begin{array}{l}\text { Generally educated in the 21st century: The importance of computer literacy in } \\
\text { an undergraduate curriculum. }\end{array}$ \\
\hline 4 & Developing and validating of a perceived ICT literacy scale for junior \\
\hline
\end{tabular}


VI Congresso Brasileiro de Informática na Educação (CBIE 2017)

Anais do XXIII Workshop de Informática na Escola (WIE 2017)

\begin{tabular}{|c|c|}
\hline $\mathbf{N}^{\mathbf{o}}$ & Artigos \\
\hline & secondary school students: Pedagogical and educational contributions. \\
\hline 5 & Using the Internet: Skill related problems in users' online behavior. \\
\hline 6 & Away with computer literacy modules at universities, or not? \\
\hline 7 & $\begin{array}{l}\text { Information and communication technology literacy issues in higher } \\
\text { Education. }\end{array}$ \\
\hline 8 & $\begin{array}{l}\text { Minimum computer literacy at tertiary institutions - Who's responsibility, is } \\
\text { it? }\end{array}$ \\
\hline 9 & $\begin{array}{l}\text { A survey of the computer literacy of undergraduate dental students at a } \\
\text { University Dental School in Ireland during the academic year 1997-98. }\end{array}$ \\
\hline 10 & Bringing computer literacy into perspective. \\
\hline
\end{tabular}

No quadro 4 estão expostos os artigos considerados no ERIC:

Quadro 4. Artigos considerados no ERIC

\begin{tabular}{|c|l|}
\hline $\mathbf{N}^{\mathbf{0}}$ & \multicolumn{1}{|c|}{ Artigos } \\
\hline 1 & $\begin{array}{l}\text { The Impact of the Students' Gender and High School Type on the Level of Digital } \\
\text { Competency Development for Lifelong Learning. }\end{array}$ \\
\hline 2 & $\begin{array}{l}\text { Software Literacy and Student Learning in the Tertiary Environment: PowerPoint and } \\
\text { Beyond. }\end{array}$ \\
\hline 3 & Empowering the Non-Traditional College Student and Bridging the "Digital Divide". \\
\hline
\end{tabular}

\subsection{Síntese e análise dos artigos selecionados}

A partir da leitura dos artigos selecionados realizou-se as análises, as comparações e a síntese dos dados. Os objetivos dos artigos foram analisados e comparados com a proposta de pesquisa desta revisão. Por fim, com o resultado da leitura de cada artigo, buscou-se solução para as questões investigadas.

\section{Resultados e Análises}

Com base nos artigos obtidos com a revisão sistemática, avaliou-se dois pontos sobre a $\mathrm{AC}$, os quais estão descritos como questões de pesquisas. As discussões sobre essas questões de pesquisa estão apresentadas a seguir.

\subsection{Questão de Pesquisa 1 - QP1}

Esta questão de pesquisa é voltada para a medição do nível de Alfabetização Computacional (AC) dos indivíduos. Kegel e Wieringa (2016) mediram o nível de AC 
por meio do comportamento de navegação, da velocidade de digitação, e da atividade de instalação. Esses autores destacam que a medição da $\mathrm{AC}$ atende a uma dupla finalidade: permite a comunicação com o utilizador num nível tecnicamente apropriado e auxilia na avaliação de riscos a que um utilizador está sujeito, tais como, phishing e vírus. Devido a essas questões, os autores implementaram um sistema que coleta medições relacionadas à informática. Essas medições incluem: comportamento de navegação, velocidade de digitação e atividade de instalação.

Por outro lado, Deursen e Dijk (2009) utilizaram alguns itens de operação relacionados ao navegador. De acordo com esses autores, para medir as competências operacionais da internet, são necessárias as seguintes operações em um navegador: (1) abrir sites digitando a URL na barra de localização; (2) navegar para frente e para trás entre as páginas, usando os botões expostos pelo navegador; (3) salvar arquivos no disco rígido; (4) abrir vários formatos de arquivo comum (ex.: PDF); (5) acessar sites de bookmarking; e (6) mudar as preferências do navegador. Por fim, cabe destacar que Shannon (2008) trata isso de uma outra forma. Ele utiliza uma escala Likert [Likert 1932] de cinco pontos, medindo as competências dos indivíduos variando de "sem conhecimento" até o nível de "Expert".

Com base nos trabalhos mencionados nessa questão de investigação, percebeu-se que a maioria dos autores mediu o nível de $\mathrm{AC}$ por meio de comportamento e operações em um navegador, visto que essas ações são muito utilizadas pelos indivíduos; nenhum desses, por exemplo, mencionou a medição por meio de editores de texto, editores de planilhas, dentre outros quesitos que são importantes e que são utilizados diariamente pelos indivíduos.

Da mesma forma, o trabalho de Wang e Prado (2015) mostrou a importância do navegador, identificando que a conectividade atualmente é o fator de maior relevância da AC. Os autores mencionaram que uma das fragilidades do processo de ensino da AC no ensino médio, em especial público, é em relação a conectividade, resultando em um acesso à internet de baixa qualidade, dificultando o desenvolvimento adequado de programas de ensino.

\subsection{Questão de Pesquisa 2 - QP2}

Esta questão de pesquisa retrata quais os instrumentos existentes para avaliar o grau de AC de indivíduos. Kegel e Wieringa (2016) implementaram um sistema de medição da AC com o intuito de personalizar as interações com o usuário. Esses autores utilizaram um sistema para medir a construção teórica com base em definições publicadas na literatura. Foi necessário implementar um sistema de medição da AC com o objetivo de utilizar medições para personalizar as interações com o usuário. Concluiu-se que a AC influencia a percepção da tecnologia por parte do usuário, bem como a compreensão dessa associada a qualquer mensagem relacionada ao computador.

Diversos instrumentos relacionados à avalição do conhecimento em computação foram identificados na literatura, como destacado a seguir. Alguns foram aplicados de forma anônima, outros ainda com tempo pré-definido para serem respondidos com amostras maiores de alunos.

- Lau e Yuen (2015) criaram um instrumento de pesquisa online. Trata-se de um questionário auto relatado sobre o uso de TIC com questões relacionadas a AC 
percebidas pelos alunos. Os alunos que testaram o instrumento foram obrigados a terminar a pesquisa durante a aula em 20 minutos.

- Os trabalhos de Deursen e Dijk (2009) e Lau e Yuen (2014) são bem semelhantes ao anterior. Eles criaram um instrumento de pesquisa online no qual os alunos foram convidados a responder.

- Dednam (2009) elaborou um questionário, o qual foi distribuído para os alunos de informática básica responderem, como parte da primeira atribuição prática. Foram feitas perguntas sobre a experiência dos alunos com programas de computador, tais como, o MS Word, além de questões sobre internet e outros tópicos, que estavam incluídas no módulo básico de AC.

- Ray e Hannigan (1999) também usaram um questionário. Porém esse foi aplicado de forma anônima em uma palestra. Os estudantes foram informados de que o questionário não era um "teste" e que nenhum crédito seria obtido por meio dele.

- Grncharovska (2016) elaborou um questionário com escala de avaliação que foi aplicado em 160 alunos do ensino médio do município de Tetovo. Os resultados obtidos mostraram que os alunos são capazes de usar a tecnologia digital quando se trata de tarefas mais simples, porém é necessário mais trabalho para desenvolver a competência digital.

- Khoo (2014) criou uma pesquisa online para coletar os dados referentes ao nível de AC dos indivíduos, que considerou as interações entre eles. Os dados foram coletados por meio de uma pesquisa on-line de estudantes (179 entrevistados) e de grupos focais de estudantes (36 participantes). A análise dos dados foi sustentada pela teoria sociocultural, que direcionou a atenção para as interações entre as pessoas, as ferramentas que usam para atingir propósitos específicos e os cenários em que as interações ocorrem.

Com base nas discussões apresentadas pelos autores, é notório que não há diversidade de instrumentos existentes para avaliar o grau de $\mathrm{AC}$ de indivíduo. A grande maioria dos instrumentos identificados na literatura são baseados em questionários (online ou não) para avaliação esses indivíduos, mudando apenas a forma com a qual se aplica. É visto que não existe uma preocupação em elaborar um instrumento que efetue uma avaliação utilizando os recursos de computação que efetivamente o indivíduo tem que usar, como por exemplo, elaborar um instrumento que possa avaliar o aluno fazendo uso de notebook, desktop, smartphone, ou seja, usando equipamentos relacionados à computação que avaliem o desempenho deles em situações reais do dia a dia.

\section{Considerações finais}

A partir da análise de diversos artigos sobre AC pode-se consolidar um conhecimento sobre o tema. Foram identificados as formas de avaliação do nível de Alfabetização Computacional (AC) e os instrumentos utilizados para essa avaliação.

Este estudo mostrou que há diversos instrumentos na literatura que mediram a AC. Alguns com características diferentes, em relação ao anonimato, à forma de aplicação, ao tempo máximo para responder ao instrumento, entre outras. Outro aspecto 
que pôde ser evidenciado foi a mudança nos instrumentos conforme a evolução tecnológica ocorre. Tópicos que em uma determinada época representavam conhecimento necessário para se considerar um indivíduo computacionalmente alfabetizado, atualmente não é mais necessário. Da mesma forma, novos conhecimentos que surgiram em função de novas tecnologias, se tornaram necessários. Basicamente a evolução da internet e a conectividade foram fatores que provocaram uma mudança nos instrumentos de avaliação da AC.

Como trabalho futuro, pretende-se dar continuidade ao estudo da AC. A proposta é elaborar um instrumento de avaliação da $\mathrm{AC}$, a partir desta revisão sistemática da literatura, para avaliar indivíduos que estão concluindo o ensino médio e iniciando um curso universitário ou se dirigindo ao mercado de trabalho.

\section{Referências}

Aureliano, V. C. O. and Tedesco, P. C. A. R. (2012). "Ensino-aprendizagem de programação para iniciantes: uma Revisão Sistemática da Literatura focada no SBIE e WIE". Brazilian Symposium on Computers in Education (Simpósio Brasileiro de Informática na Educação - SBIE), vol. 23, n. 1.

Costa, C. S. (2010). "Uma abordagem baseada em evidências para o gerenciamento de projetos no Desenvolvimento distribuído de software". Dissertação de Mestrado, UFP, 170p.

Coutinho, C. P. and Lisbôa, E. S. (2011). "Sociedade da informação, do conhecimento e da aprendizagem: desafios para educação no século XXI". Revista de Educação, 18(1): 5-22.

Dednam, E. (2009). “Away with computer literacy modules at universities, or not?” In: Proceedings of the 2009 Annual Conference of the Southern African Computer Lecturers' Association, p. 23-32, ACM.

Deursen, A. J. V. and Dijk, J. A. V. (2009). "Using the internet: skill related problems in users' online behavior”. Interacting with computers, Elsevier, v. 21, n. 5, p. 293-402.

Filatro, A. and Piconez, S. C. B. (2014). "Design instrucional contextualizado-educação et". Senac.

Grncharovska, S. P. et al. (2016). "The impact of the students' gender an high school type on the level of digital competency development for lifelong learning". Journal of Education an Practice, v. 7, n. 24, p. 108-114.

Gupta, G. K. (2006). “Computer Literacy: Essential in today's computer-centric world”. ACM SIGCSE Bulletin, ACM, v. 38, n.2, p. 115-119.

Kegel, R. H. P. and Wieringa, R. J. (2016). "Measuring computer literacy without questionnaires". p. 61-65.

Khoo, E., Hight, C., Cowie, B., Torrens, R. and Ferrarelli, L. (2014). "Software literacy and student learning in the tertiary environment: PowerPoint and beyond". Journal of Open, Flexible and Distance Learning, 18(1): 30-45.

Kitchenham, B. A. (2007). "Guidelines for performing Systematic Literature Reviews in Software Engineering”. 
VI Congresso Brasileiro de Informática na Educação (CBIE 2017)

Anais do XXIII Workshop de Informática na Escola (WIE 2017)

Lau, W. W. and Yuen, A. H. (2014). "Developing and validating of a perceived ict literacy scale for junior secondary school students: Pedagogical and educational contributions". Computers \& Education, Elsevier, v. 78, p. 1-9.

Lau, W. W. and Yuen, A. H. (2015). "Factorial invariance across gender of a perceived ict literacy scale". Learning and Individual Differences, 41:79-85.

Liao, L. and Pope, J. W. (2008). "Computer Literacy for everyone". Journal of Computing Sciences in Colleges, v. 23, n. 6, p. 231-238.

Moura, E. M. S. (2010). "Inclusão, alfabetização e fluência digital". Trabalho de conclusão de especialização (Pós-Graduação em Mídias na Educação) Universidade Federal do Rio Grande do Sul, Porto Alegre.

Likert, Rensis. (1932). "A technique for the measurement of attitudes". Archives of Psychology 140: pp. 1-55.

Ray, N. J. and Hannigan, A. (1999). "A survey of the computer literacy of undergraduate dental students at a university dental school in Ireland during the academic year 1997-98”. European journal of dental education, 3(2):56-63.

Shannon, L. (2008). "Information and communication technology literacy issues in higher education". Information Systems Education Journal, 6(23):3-13.

Wang, M. A. and Prado, E. P. V. (2015). "Critérios para avaliação da alfabetização computacional”. Dissertação (Mestrado) - Universidade de São Paulo. 